\title{
Analysis of the white-light flickering of the intermediate polar V709 Cassiopeiae with wavelets and Hurst analysis
}

\author{
F. Tamburini ${ }^{1}$, D. de Martino ${ }^{2}$, and A. Bianchini ${ }^{1}$
}

\author{
1 Department of Astronomy, University of Padova, vicolo dell' Osservatorio 3, 35122, Padova, Italy \\ e-mail: fabrizio.tamburini@unipd.it \\ 2 INAF-Astronomical Observatory of Capodimonte, Moiarello 16, 80131, Naples, Italy \\ Received 13 January 2009 / Accepted 12 April 2009
}

\section{ABSTRACT}

\begin{abstract}
We characterize the flickering observed in the optical light curve of the intermediate polar system V709 Cas by determining its position in the $\alpha-\Sigma$ diagram of Fritz and Bruch classification scheme. The strength of flickering on a given timescale is represented by $\Sigma$, while $\alpha$ is the energy distribution of the flickering at different timescales. Here $\alpha$ is derived independently with both the wavelets and the Hurst $R / S$ analysis. The flickering shows self-similarity on a timescale ranging from tens of minutes down to $10 \mathrm{~s}$ with stochastic persistent memory in time. The parameters $\alpha$ and $\Sigma$ appear to be anticorrelated. In the $\alpha-\Sigma$ diagram, V709 Cas occupies in the region of magnetic systems. Since V709 Cas shows the spin period of the magnetic WD only in the X-ray but not in the optical, we conclude that this method can be used to characterize CV subtypes especially when their classification is uncertain.
\end{abstract}

Key words. stars: binaries: close - accretion, accretion disks - chaos

\section{Introduction}

Cataclysmic variables (CVs) are binary systems in which a latetype secondary star fills its Roche lobe and transfers matter onto a white dwarf (WD) primary. The WD is surrounded by an accretion disk, unless its magnetic field is strong enough to partially or totally control the accretion geometry.

CVs are classified into three major groups: nova-like (NL) systems, classical and recurrent novae $(\mathrm{CN}, \mathrm{RN})$, and dwarf novae (DN). In particular, nova systems must host CNO WDs with higher masses than $\sim 0.6 \quad M_{\odot}$ so that the accreted material can be cyclically ejected through nova outbursts (Livio 1992). We can also classify CVs according to the strength of the magnetic field of the primary. Thus, we have the non-magnetic systems, the Intermediate Polar systems or DQ Her systems (hereafter IPs), and the Polars or AM Her systems. In the non-magnetic systems, accretion onto the WD occurs from the last stable orbit of the inner disk. In IP systems, the magnetic field disrupts the inner regions of the accretion disk within the so-called Alfvén radius and accretion occurs mainly via accretion curtains onto the magnetic poles of the WD. In polar systems, the disk is totally disrupted by the strong magnetic field of the WD and the accretion stream is directly conveyed on the WD magnetic poles. In both polars and IPs, the infalling matter forms a strong shock above the magnetic poles of the compact star that mainly radiate in the X-ray domain and eventually produce cyclotron radiation in the optical/IR.

At any given orbital period, the luminosities of NL systems and quiescent novae are systematically brighter than those of $\mathrm{DN}$, suggesting that the former are powered by higher mass transfer rates $\left(\dot{M}_{2}\right)$ from the secondary and/or have hotter WDs preventing the onset of the cyclical disk instability phenomena observed in DN. An exception may be represented by those $\mathrm{CN}$ and NL that are also magnetic polars in which the strong magnetic field of the WD reduces the efficiency of the magnetic braking mechanism of the binary system and, consequently, the mass transfer rate and the accretion luminosity (Warner 1995).

The light curves of CVs may exhibit a variety of periodic, quasi-periodic, and/or erratic modulations. Flickering consists of short-term variations in brightness on timescales from a few seconds to a few tens of minutes, of between a few tenths of magnitude and one magnitude. Flickering appears as a sequence of overlapping flares and bursts with a random variability in time, sometimes exhibiting self-similarity on different timescales, i.e., the stochastic fluctuations in the detrended light curve are similar independently from the chosen time binning. Warner \& Nather (1971) first tried to associate the flickering of U Gem and DN with the hot-spot in the outer rim of the accretion disk, although this ansatz was not confirmed in the observations of some other CVs such VW Hyi (Warner 1975; van Amerongen et al. 1987), Z Cha (Bruch 1996), V2051 Oph (Warner \& O'Donoghue 1987). The observations of AE Aqr and YZ Cnc (Elsworth \& James 1982, 1986; James 1987), instead, suggested that the origin of the flickering might be in the innermost regions of the accretion disk or close to the surface of the accreting WD. Some models suggested that the instabilities in the mass accretion onto the WD (Bruch 1992a,b,c, 1996) are caused by magneto-hydro-dynamical plasma turbulence in the accretion flow. The flickering of CVs was studied by using high speed photometry campaigns (see, for example, the works of Ribeiro \& Diaz (2007) and Baptista \& Bortoletto (2004)).

Flickering has been observed in both non-magnetic and magnetic CVs. Magnetic CVs usually exhibit flickering in the optical and sometimes the X-ray region of the spectrum. One general property of flickering is the correlation between its amplitude and the luminosity of the "quiet primary". The "quiet primary" is defined as the sum of the luminosity of the WD and the luminosity of that part of the accretion process (disk or flow) that is not involved in the flickering activity (Zamanov \& Bruch 1998). Information can also be obtained by comparing the flare rate and the ratio of the maximum to the mean flux observed with those of 
the quiet primary. In some objects, the flickering involves a large fraction of the total light emitted eventually dominating the optical light curve. The modalities of the flickering observed may vary from object to object but some common features have been observed in CVs of the same sub-type. Fritz and Bruch (1998) proposed a classification method of the flickering showing that different subtypes of CVs tend to occupy specific regions of the $\alpha-\Sigma$ plane, where $\alpha$ is the parameter related to the energy distribution of the flickering on different timescales and $\Sigma$ represents the strength of the flickering on a given timescale.

In this paper we derive the $\alpha$ parameter for the IP system V709 Cas using both the wavelet and the Hurst $R / S$ analyses (Hurst et al. 1965) and discuss the two methods. While the wavelet analysis of flickering is dependent on the choice of the mother wavelet function, Hurst's $R / S$ analysis bypasses this problem, providing an independent determination of $\alpha$. In addition, the value of the Hurst exponent indicates the degree of persistence/anti-persistence of the stochastic memory of the flickering, depicting the global behavior of the physics of the accretion process. In a different way, from the X-ray band, this magnetic $\mathrm{CV}$ shows no spin modulation in the optical range and it is strongly flickering-dominated, without any precise and stable dominating frequency. V709 Cas hence represents an ideal test case for our study.

In Sect. 2 we introduce the mathematical basis for both wavelets and Hurst's analysis and explain the link between these two methods. In Sect. 3 we characterize the flickering of this IP system by placing it in the $\alpha, \Sigma$ diagram. Section 4 presents our conclusions.

\section{Flickering properties and Hurst exponent}

Many authors have discussed flickering mainly in terms of statistical properties (Mumford 1996; Robinson 1973; Moffet \& Barnes 1974; Zuckermann 1971; Bruch 1992a,b,c, 1996; Bruch \& Grütter 1997). A further step was made by Fritz \& Bruch (1998) who, following Scargle et al. (1993), introduced the use of wavelet transforms and the scalegram. The scalegram is a plot of the logarithm of a reference timescale $t_{s}$ versus the logarithm of the variance $S\left(t_{s}\right)$, namely a measure of the variances of the wavelet coefficients expressed as a function of the timescale (see also Percival \& Walden (2000) for more details).

Flickering is a stochastic process in time, whose properties can provide vital information about the driving mechanism behind the accretion process. One of the most important statistical properties of flickering is the measure of the variance in the wavelet coefficients on different timescales

$S^{\prime}\left(t_{s}\right)=\frac{2^{s}}{N} \sum_{k} c_{s, k}^{2}$

where $c_{s, k}^{2}$ are the wavelet coefficients in which $k$ is the time index, $N$ the number of measurements (equivalent to the total number of the wavelet coefficients), and $s$ is the scale index, also called "octave", related to the timescale by $t_{s}=2^{\mathrm{s}} \Delta t$. The quantity $S^{\prime}\left(t_{s}\right)$ is related to the total energy of the signal calculated on the timescale $t_{s}$ and summed over all the time bins, then normalized to both $\mathrm{N}$ and in the integration interval $\Delta t$. The function $S^{\prime}\left(t_{s}\right)$ characterizes the statistical variations in the data record in the time interval $\left[2^{s} \Delta t, 2^{s+1} \Delta t\right]$.

Fritz \& Bruch (1998) and Zamanov \& Bruch (1998) showed that the scalegrams of flickering are approximately linear functions of $t_{s}$ for most of the CVs' light curves. This implies the presence of self-similarity with a power-law behavior
$S^{\prime}\left(t_{s}\right) \sim s^{\alpha}$ on certain timescales. The stochastic fluctuations in the detrended light curve are similar independently from the time binning as described by $1 / f^{\beta}$ noise, where $f$ is the time frequency and $\beta=\alpha / 2$.

The linearity of the scalegrams permits a simple parametrization of the flickering properties with two parameters, $\alpha$ and $\Sigma$. The parameter $\alpha$ is the inclination of the scalegram with respect to the $x$ axis, which can be determined by a linear least squares fit to the scalegram points of the $\log S\left(t_{s}\right), \log \left(t_{s}\right)$ plot the parameter indicates whether slow or rapid light fluctuations dominate the stochastic time series, and the flickering strength parameter $\Sigma=\log S\left(t_{s}\right) \cdot t_{s}$, given by

$$
S\left(t_{s}\right)=S^{\prime}\left(t_{s}\right) \frac{N \Delta t}{\sum_{s, k} c_{s, k}^{2}},
$$

represents a measure of the variance in the wavelet coefficients on a given timescale. Some possible correlations between the strength and the duration of the stochastic light variations are evident by plotting $\alpha$ versus $\Sigma$ in the so-called $\alpha-\Sigma$ parameter space.

An alternative robust statistical tool that can characterize independently from the wavelet approach the stochastic properties of flickering (i.e., the parameter $\alpha$ ) is Hurst's $R / S$ analysis. This method offers the advantage of being independent of any previous modeling of the problem, such as the choice of a mother wavelet. The approach initially used by Hurst (1951) and Hurst et al. (1965) is based on the rescaled range analysis, which consists of estimating the ratio of the range in the variations, $R$, to the standard deviation $S$ derived from the analysis of all the subintervals of data for each equal partitioning of the full data record. Mandelbrot \& van Ness (1968) and Mandelbrot \& Wallis (1969) linked this method to a particular class of self-similar random processes, called Fractional Brownian Motions (FBMs). It was also shown that the short-run statistical dependence of pseudo-random sequences can be described with this technique (Gammel 1997). Hurst found that natural phenomena follow the empirical power law

$R / S=(\epsilon N)^{H}$

where $\epsilon$ is a constant, $N$ the number of data points present in each sub-interval, and $H$ is the so-called Hurst's exponent. In particular, Hurst found that purely stochastic phenomena present a mean value $H \sim 0.73$ and a standard deviation $S \sim 0.09$.

In dynamical systems, $H$ characterizes the stochastic memory in time of the process. Hurst exponents $H>\frac{1}{2}$ indicate the persistence in time of a certain trend, whereas exponents $H<\frac{1}{2}$ indicate anti-persistence, i.e., past trends tend to reverse in the future (Feder 1988). An exponent $H=\frac{1}{2}$ would then represent random uncorrelated behaviors with no stochastic memory in time. The value $H \sim 0.73$ suggests that natural phenomena tend to present a persistent stochastic memory in time.

In astronomy, Hurst's analysis has been applied mainly to solar phenomena. Most of the solar phenomena seem to have $H>\frac{1}{2}$, even if the true presence of long - memory in solar activity is still a question of debate (Oliver \& Ballester 1998). In the Sun, some physical processes also exhibit different stochastic behaviors. Hanslmeier et al. (2000), showed that fluctuations in the velocity fields and the intensity fields observed in the solar upper photosphere have $H=\frac{1}{2}$.

We consider a practical illustration of Hurst's $R / S$ analysis. The light curve of a $\mathrm{CV}$ is a discrete record in time of light intensity values that usually exhibits both slow and rapid stochastic luminosity variations. Some periodic variations are 
connected to either the orbital motion or the spin of the magnetic white dwarf. Besides these, CVs can also present quite a variety of irregular or quasi-periodic light oscillations. The shorter timescales of the flickering observed in the light curves of ordinary $\mathrm{CV}$ s are on the order of a few seconds. However, one can also observe smooth modulations and even flares lasting a few hours. The mean luminosity $\langle\xi\rangle_{\tau}$ of the $\mathrm{CV}$ in a given time interval $\tau$ is

$\langle\xi\rangle_{\tau}=\frac{1}{\tau} \sum_{t=1}^{\tau} \xi(t)$

where $t$ is the time coordinate. In the time lag $\tau$, the accumulated deviation from the mean luminosity is

$X(t, \tau)=\sum_{k=1}^{t}\left[\xi(k)-\langle\xi\rangle_{\tau}\right]$

and the range $R$ of the luminosity variations is

$R(\tau)=[\max X(t, \tau)-\min X(t, \tau)]_{1 \leq t \leq \tau}$.

As already stated, the ratio $R / S$ follows an empirical law $R / S \sim$ $\tau^{H}$, as in Eq. (3).

Depending on the value of $H$ found, the behavior of the random fluctuations can be modeled by Wiener stochastic processes or, more specifically, by Fractional Brownian Motions (FBM). The classical Brownian motion is a particular case of FBM, with $H=\frac{1}{2}$, without any stochastic memory in time (for more details, see Feder 1988). Since FBMs possess self-similarity, they can also be easily studied with the wavelet analysis (Chui 1992; Gill \& Henriksen 1990; Simonsen et al. 1998). Using the relationships between $1 / f^{\beta}$ noises and FBMs (Lowen 1999; Gao et al. 2003), there is a direct relationship between the Hurst exponent $H$ and the parameter $\alpha$, namely $H=1-\alpha / 4$.

In Fig. 1, we show the histogram of the averaged Hurst exponent of each single CV analyzed by Fritz and Bruch (1998) and reported in their Table 1. We see from this small sample of about $70 \mathrm{CVs}$ that both persistent and antipersistent behaviors are present. We note that the main peak, around $H \sim 0.68$, is preceded by another lower peak at $H=0.5$, corresponding to the case of a classical Brownian Motion. The main peak is then followed by a drastic drop around the value $H \sim 0.70$. In the neighborhood of the value $H \sim 0.73$ found by Hurst in most natural phenomena, there is a second small bump. We note that the distribution is asymmetric with a longer tail towards the lower values of $H$. A study of a large number of CVs might determine other important details about the accretion phenomena.

\section{The flickering in white light of V709 Cas}

V709 Cas (RX J0028.8+5917) is a DQ Her-type system of orbital period 0.2225 days (Bonnet-Bidaud et al. 2001) and a $1.08 M_{\odot}$ primary (Ramsay 2000) of spin period $P_{\text {spin }}=312.8 \mathrm{~s}$ (de Martino et al. 2001). We analyzed six runs obtained in white light during a multi-site photometric campaign performed between September 22 and October 2, 2000 by de Martino et al. (2002) with a three-channel photometer mounted on the $0.8 \mathrm{~m}$ Tenerife (Spain), at the $1.5 \mathrm{~m}$ Loiano (Italy) and at the $0.8 \mathrm{~m}$ Beijing (China) telescopes. The integration time in all cases was $10 \mathrm{~s}$.

The low frequency trend was removed by subtracting a $625 \mathrm{~s}$ smoothing average. Figure 2 shows the very rapid variations of up to $0.2 \mathrm{mag}$, indicating the presence of a strong flickering, already reported by Kozhevnikov (2001) and de Martino et al. (2002). Each inset of Fig. 2 shows the "corrected light curve",

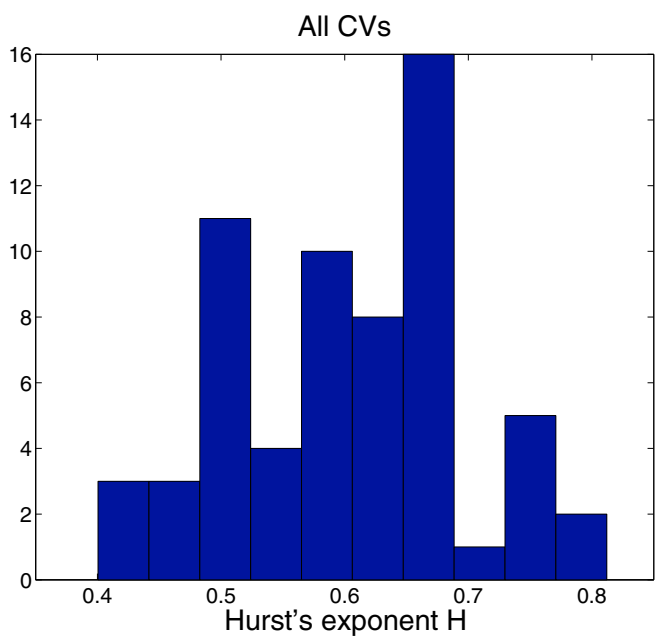

Fig. 1. Histogram of the averaged Hurst exponent of all the sample of CVs analyzed by Fritz \& Bruch 1998, (reported in their Table 1). The distribution is asymmetric showing a tail towards the shorter values of $H$ and peaks at $H \simeq 0.68$.

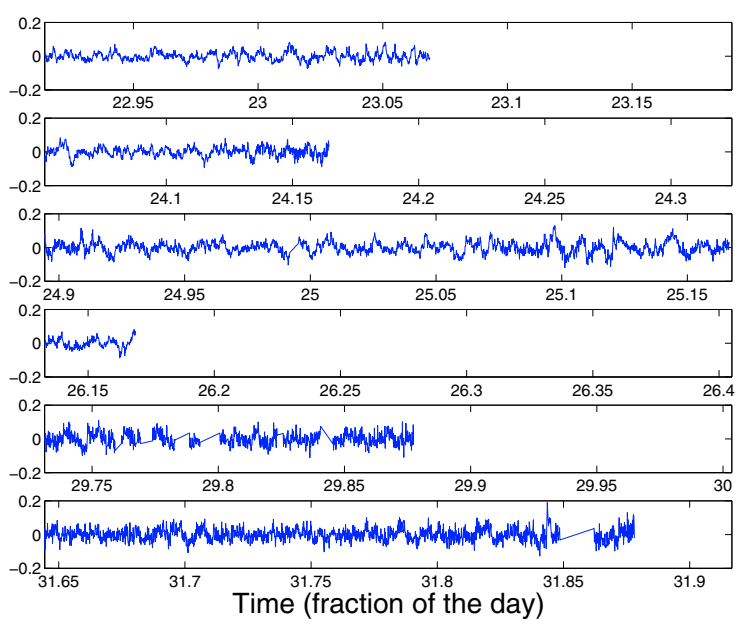

Fig. 2. Corrected light curves of the six runs (a-f) of Table 1. Time is in days and magnitudes are rescaled with respect to their mean magnitude. The discontinuities in the last two datasets are caused by the presence of clouds during observations.

i.e. the light-curve variations in magnitude rescaled to zero and filtered of fluctuations due to the orbital period of the CV. The data sets are ordered in Table 1. From top to bottom, the first subplot (a), shows, with respect to the other subsets of data, a more active flickering, with more erratic motions, rapid and intense flares. The third and fifth subplot (c) and (f) show a larger variance on shorter timescales and smaller variance on longer timescales. They both present flares of larger amplitudes up to $0.2 \mathrm{mag}$. Power spectra of the corrected light curves do not show any dominating periodicity (Fig. 3). The presence of some residual power $\left(\sim 10^{-4}\right)$ is present at low frequencies, but does not appreciably affect the results of our analysis.

\subsection{Data analysis}

We now determine the parameter $\alpha$ by using both wavelets and a Hurst $R / S$ analysis, and for each run plot the position of V709 Cas in the parameter space $\alpha-\Sigma$ with the timescale resolution of 
Table 1. From first column: label, start, end of the run, number of data points, max, min mag., characteristic exponent $\beta$, Hurst exponent $H$, and percentage difference $\Delta \%$ (see text).

\begin{tabular}{|c|c|c|c|c|c|c|c|c|}
\hline Run & Start $(\mathrm{dd})^{\dagger}$ & Stop (dd) & $\begin{array}{l}\text { number } \\
\text { of data }\end{array}$ & $\begin{array}{l}\text { Max } \\
\text { mag }\end{array}$ & $\begin{array}{l}\min \\
\operatorname{mag}^{\ddagger}\end{array}$ & $\bar{\beta}$ & $\bar{H}$ & $\overline{\Delta \%}$ \\
\hline $\mathrm{a}$ & 0.414402 & 0.568802 & 1335 & 0.082 & -0.075 & 0.82 & 0.59 & 1.2 \\
\hline b & 2.466237 & 2.578852 & 974 & 0.084 & -0.093 & 0.72 & 0.64 & 8.3 \\
\hline $\mathrm{c}$ & 3.308728 & 3.581072 & 2325 & 0.130 & -0.119 & 0.80 & 0.60 & 3.8 \\
\hline d & 4.547223 & 4.583219 & 312 & 0.081 & -0.087 & 0.56 & 0.72 & 4.3 \\
\hline $\mathrm{e}$ & 8.145587 & 8.291772 & 1029 & 0.108 & -0.111 & 0.72 & 0.64 & 4.2 \\
\hline \multirow[t]{2}{*}{$f$} & 10.058795 & 10.292484 & 1904 & 0.190 & -0.127 & 0.82 & 0.59 & 7.3 \\
\hline & $\begin{array}{l}\text { from JD } \\
2451810\end{array}$ & $\begin{array}{l}\text { rescaled } \\
\text { to mag } 0\end{array}$ & & & & & & \\
\hline
\end{tabular}

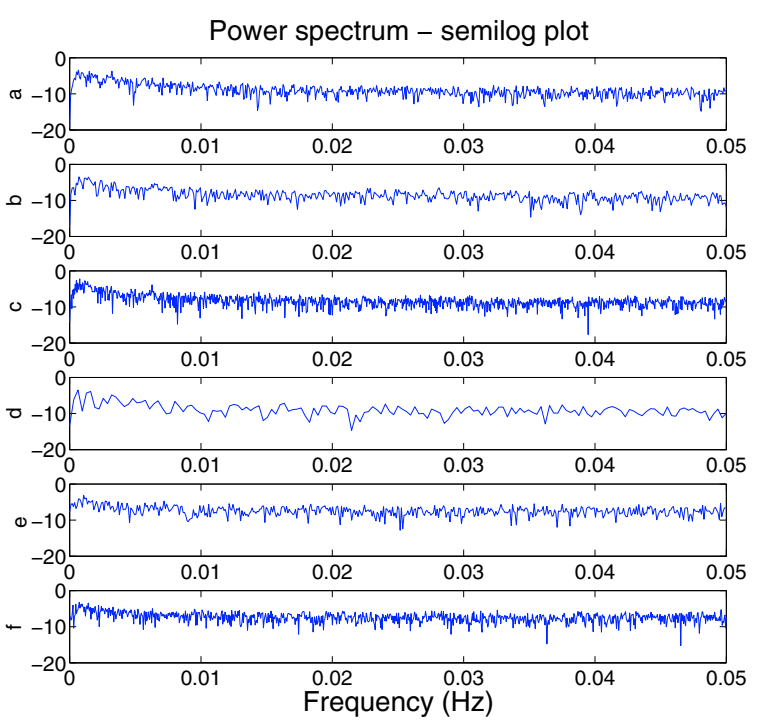

Fig. 3. Semilog-plot of the power spectra of the six corrected light curves of V709 Cas.

10 s. We used the C12 wavelets (Fritz \& Bruch 1998). To test our software, we used both wavelet-generated FBMs and $1 / f^{\beta}$ Gaussian processes (Bak et al. 1987; Abry et al. 1995). Each light curve (a-f) was divided into series of $2^{n}$ bins, where $n$ is an integer that varies in the interval $[1,7]$ to apply the prescriptions of the wavelet analysis.

For each run, Table 1 gives the characteristic exponent $\beta$ of the $1 / f^{\beta}$ process obtained with wavelets and the Hurst exponent $H$ with an $R / S$ analysis. The table also reports the run label, the number of data points, the maximum and minimum of the relative magnitudes, and the percentage difference $\Delta \%=$ $\frac{\left|D_{H}-D_{f}\right|}{\left|D_{H}+D_{f}\right|} \times 100$ between the fractal exponent $D$ calculated with either the $1 / f^{\beta}$ noises $\left(D_{f}=1+\beta / 2\right)$ or the $R / S$ analysis $\left(D_{H}=2-H\right)$. The start of each run is calculated in number of days from the starting Julian day 2451810.

The Hurst exponents of each of the six acquisitions reported in Table 1 are all higher than $1 / 2$, indicating that the flickering of V709 Cas is always a stochastic process with a persistent memory in time. The averaged value of $H$ is 0.63 and the standard deviation is 0.05 . The value obtained with $R / S$ the analysis, reported in the table, fluctuates between $H=0.58$ and $H=0.72$. However, the differences $\Delta \%$ are rather small. Figure 4 presents the scalegrams for the six corrected light curves, i.e., the log$\log$ plot of the variance $S\left(t_{s}\right)$ versus the chosen timescale $t_{s}$. In

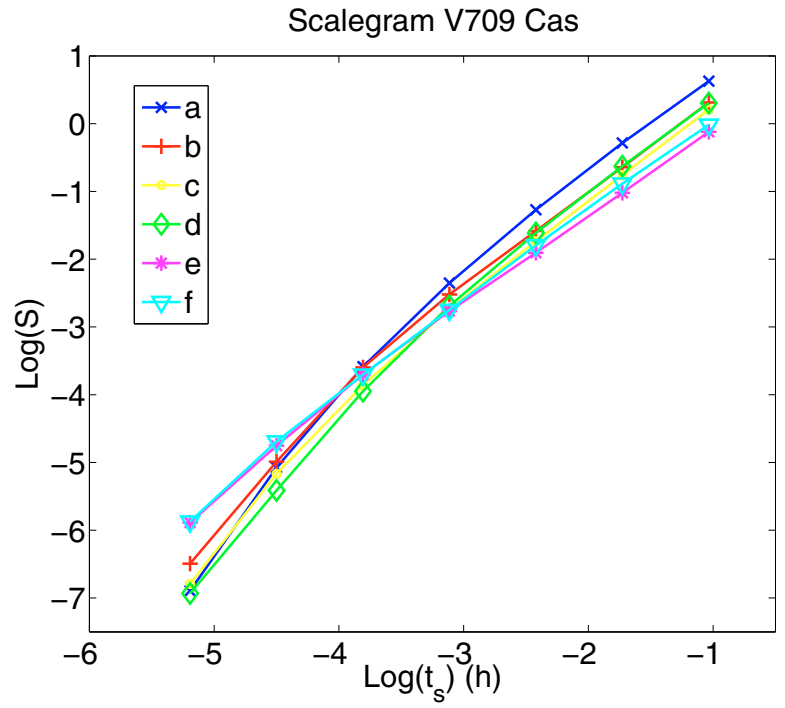

Fig. 4. Scalegrams of the six acquisitions of the corrected V709 Cas data. The lines corresponding to different acquisitions are quite similar illustrating the stability of $\alpha$ over 10 days. The small differences on short timescales may be due to different Poisson noise contributions. Here and in Fig. 5 we adopted the same colors (online) and symbols for each different run

Figs. 4 and 5 we adopted the same colors (in the online edition) and symbols to label each different run.

The curves rise linearly with the time intervals and remain quite linear for all the 6 acquisitions. This suggests that the flickering remains self-similar with the same power-law exponent, from tens of minutes down to a $10 \mathrm{~s}$ timescale. We also see that all the curves almost overlap yielding a quite stable value of $\alpha$, and indicating that, during the 10-day period of observations, the flickering and the accretion process of the $\mathrm{CV}$ remained quite stable.

Figure 5 shows the $\alpha$ and $\Sigma$ diagram of V709 Cas. In the plot we decided only to draw, for each run, the relevant values of each wavelet octave to illustrate the erratic motion of the CV in the parameter space. We note that, while almost all the runs exhibit erratic motion in the $\alpha-\sigma$ plane, the run $\mathrm{f}$ shows a very high stochastic stability, being always confined around the values $\alpha \sim 1.6$ and $\Sigma \simeq-0.8$ (reported in the plot). The values span the intervals $0.9<\alpha<1.8$ and $-1.3<\Sigma<-0.6$, which are typical of magnetic CVs (Bruch 1992a). In the $(\alpha, \Sigma)$ plane, the values obtained for V709 Cas show a trend. The parameter $\Sigma$ is shown to decrease with decreasing $\alpha$. This would suggest that the light fluctuations and the energies implied are anticorrelated. 


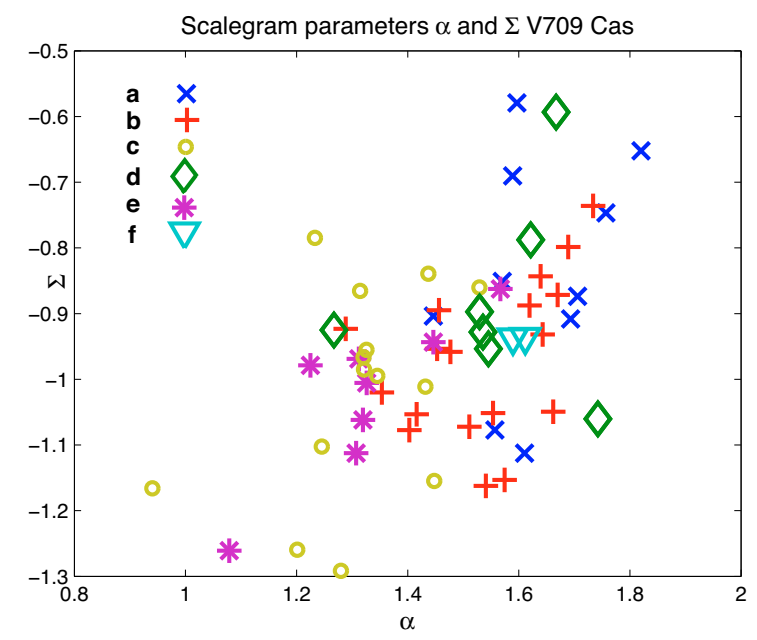

Fig. 5. $\alpha-\Sigma$ parameter space for all the V709 Cas data. Only the relevant values for each wavelet octave are reported in the plot. While almost all the runs show erratic motion in the $\alpha-\Sigma$ plane, the run f shows a very high stochastic stability, being always confined around the values $\alpha \sim 1.6$ and $\Sigma \simeq-0.8$ (reported in the plot). The relatively small region of the diagram occupied by the data indicates a rather stable stochastic phenomenon. Here and in Fig. 4, we adopted the same colors (online) and symbols for each different run.

\section{Conclusions}

We have analyzed the flickering observed in six optical photometric runs of the IP V709 Cas. In their pioneering work, Fritz $\&$ Bruch (1998) tried to correlate the characteristics of the flickering of CVs with their subtypes using a wavelet analysis and the scalegram. The stochastic properties of flickering were described by using the two parameter space $\alpha$ - $\Sigma$. In this paper, the parameter $\alpha$ was obtained both with wavelets and the Hurst $R / S$ analysis, the latter being a statistically robust method. During the 10 days of observations, the stochastic properties of the flickering did not change indicating that the physics of the accretion process remained relatively stable. We also noticed that the values of $\alpha$ and $\Sigma$ parameters, calculated at different epochs and on different timescales, show a trend suggesting that the persistence character of the light fluctuations and the energies implied in the accretion process are anticorrelated. In the $(\alpha, \Sigma)$ diagram, V709 Cas occupies the same region as magnetic systems. However, this $\mathrm{CV}$ was recognized as an IP only on the basis of its X-ray light curve, while no spin modulation in the optical range was observed and the light curve was found to be strongly flickeringdominated without any precise and stable dominating frequency, with the exception of frequencies related to the orbital motion. Therefore, V709 Cas may represent an ideal test case where the $\alpha-\Sigma$ classification improved by the Hurst $R / S$ analysis can be used to characterize CV subtypes, especially when the classification is uncertain.

Hurst's $R / S$ analysis can also provide a useful tool for understanding the results of high speed photometry. In particular, it could provide precious information to indicate down to which short timescale the flickering can still be considered self-similar, determining the physics behind very short timescale phenomena. Ultra-fast photometry, as presently achievable with ULTRACAM on the VLT and SALTICAM on SALT, which can achieve a time resolution lower than $100 \mathrm{~ms}$ are ideally suited to studying rapid variability in CVs. These observations will significantly improve this investigation as illustrated by the results from a high-speed photometric survey of faint CVs with a separate focus on periodic/quasi-periodic variability in CVs made with SALT (Warner \& Woudt 2008). A futuristic application might be ultra-fast photometry towards the quantum limit proposed for overwhelming large telescopes (Dravins et al. 2005).

Acknowledgements. One of us, F.T., also gratefully acknowledges the financial support from the CARIPARO Foundation inside the 2006 Program of Excellence. D.d.M. and A.B. acknowledge financial support from INAF PRIN N.17. The authors are grateful to Patrick Woudt for his stimulating comments and corrections.

\section{References}

Abry, P., Gonçalvès, P., \& Flandrin, P., in Wavelets and Statistics, ed. A. Antoniadis, \& G. Oppenheim (Springer-V.), Lect. Notes Stat., 103

Bak, P., Tang, C., \& Wiesenfeld, K. 1987, Phys. Rev. Lett., 59, 381

Baptista, R., \& Bortoletto, A. 2004, AJ, 128, 411

Bonnet-Bidaud, J. M., Mouchet, M., de Martino, D., Matt, G., \& Motch, C. 2001, A\&A, 374, 1003

Bruch, A. 1992a, A\&A, 266, 237

Bruch, A. 1992b, in Viña del Mar Workshop on Cataclysmic Variable Stars, ASP Conf. Ser., 29, ed. N. Vogt

Bruch, A. 1992c in Flares and Flashes, ed. J. Greiner et al. (Springer Verlag), Heidelberg, Proc. IAU Coll., 151, 288

Bruch, A. 1996, A\&A, 312, 97

Bruch, A., \& Grutter, M., Acta Astron., 47, 307

Chui, C. K. 1992, An Introduction to Wavelets (Academic Press Inc., San Diego de Martino, D., Matt, G., Mukai, K., et al. 2001, A\&A, 377, 499

de Martino, D., Silvotti, R., Gonza'lez Pe'rez, J. M., et al. 2002, ASPC, 261, 145

Dravins D., Barbieri C., Da Deppo V., et al. 2005, QuantEYE. Quantum Optics Instrumentation for Astronomy, OWL Instrument Concept Study, ESO document OWL-CSR-ESO-00000-0162

Elsworth, Y. P., \& James, J. F., 1982, MNRAS, 198, 889

Elsworth, Y. P., \& James, J. F., 1986, MNRAS, 220, 895

Feder, J. 1988, Fractals (NY: Plenum Press)

Fritz, T., \& Bruch, A. 1998, A\&A, 332, 586

Gammel, B. M. 1997, Phys. Rev. E, 58, 2, 2586

Gao, J. B., Yinhe Cao, \& Lee. J.-M. 2003, Phys. Lett. A, 314, 392

Gill, A. G., \& Henriksen, R. N. 1990, ApJ, 365, L27

Haberl, F., \& Motch, C. 1995, A\&A 297, L37

Hanslmeier, A., Kucera, A., Rybák, J., Neunteufel, B., \& Wöhl, H. 2000, A\&A, 356,308

Hurst, H. E., Trans. Am. Soc. Civ. Eng. 116, 770

Hurst, H. E., Black, R., \& Sinaika, Y. M., Long-Term Storage in Reservoirs: An Experimental Study (Constable, London)

James, J. F. 1987, MNRAS, 224, 227

Kozhevnikov, V. P. 2001, A\&A, 366, 891

Livio, M. 1992, ApJ, 393, 516.

Lowen, S. B. 1999, Methodology and Computing in Applied Probability 1, 4, 445-456, (Springer Netherlands ISSN 1387-5841 (print) 15737713 (online))

Mandelbrot, B. B., \& van Ness, 1968, SIAM (Soc. Ind. Appl. Math.) Rev., 10, 422

Mandelbrot, B., \& Wallis, J. 1969, Water Resources Res., 5, 321

Moffett, T. J., \& Barnes, T. G. 1974, ApJ, 194, 141

Mumford, G. S. 1966, ApJ, 146, 411

Norton, A. J., Beardmore, A. P., Allan, A., \& Hellier, C. 1999, A\&A, 347, 203.

Oliver, R., \& Ballester, J. L. 1998, Phys. Rev. E, 58, 5, 5650

Percival, D. B., \& Walden, A. T. 2000, Wavelet Methods for Time Series Analysis (Cambridge University Press)

Ramsay, G. 2000, MNRAS, 314, 403

Ribeiro, F. M. A., \& Diaz, M. P. 2007, AJ, 133, 2659

Robinson, E. L. 1973, ApJ, 180, 121; ApJ, 181, 531; ApJ, 183, 193

Scargle, J. D., Steinman-Cameron, T. Y., Young, K., et al. 1993, ApJ, 411, L91

Simonsen, I., Hansen, A., \& Magnar Nes, O. 1998, Phys. Rev. E, 58, 2779

van Amerongen, S., Damen, E., Groot, M., Kraakman, H., \& van Paradijs, J. 1987, MNRAS, 225, 93

Warner, B. 1975, MNRAS, 170, 219

Warner, B. 1995, Cataclysmic Variable Stars (Cambridge University Press)

Warner, B., \& Nather, R. E. 1971, MNRAS, 152, 219

Warner, B., \& O'Donoghue, D. 1987, MNRAS, 224, 733

Warner, B., \& Woudt, P. A. 2008, in COOL DISCS, HOT FLOWS: The Varying

Faces of Accreting Compact Objects, ed. M. Axelsson, AIP Conf. Proc., 1054, 101

Zamanov, R. Z., \& Bruch, A. 1998, A\&A, 338, 988

Zuckermann, M.-C. 1961, Ann. d'Astrophys., 24, 431 